\title{
Urgences
}

\section{Le cour de la baleine bleue de Jacques Poulin : de la poésie au} roman

\section{Jean Morency}

Numéro 28, mai 1990

Le roman comme poétique

URI : https://id.erudit.org/iderudit/025586ar

DOI : https://doi.org/10.7202/025586ar

Aller au sommaire du numéro

Éditeur(s)

Urgences

ISSN

0226-9554 (imprimé)

1927-3924 (numérique)

Découvrir la revue

Citer cet article

Morency, J. (1990). Le cœur de la baleine bleue de Jacques Poulin : de la poésie au roman. Urgences, (28), 30-40. https://doi.org/10.7202/025586ar d'utilisation que vous pouvez consulter en ligne.

https://apropos.erudit.org/fr/usagers/politique-dutilisation/ 


\section{Le cour de la baleine bleue de Jacques Poulin: de la poésie au roman}

\section{Jean Morency}

$\mathrm{Au}$ « cœur» de l'œuvre romanesque de Jacques Poulin, on retrouve un assez curieux personnage: le *commis aux écritures". Celui-ci ne se considère pas vraiment comme un "écrivain", bien que l'écriture tienne une grande place dans sa vie: autant l'écrivain peut être libre et magnifique, à la manière du vieux Hemingway, grand chasseur de fauves devant l'éternel, autant le commis semble voué à un apostolat des plus obscurs. Ignorant la volonté et l'énergie, il n'écrit pas: il transcrit. Attentif à des voix qui lui viennent du dedans, moins créateur que récepteur, il "travaille a à ses romans en lisant Gaston Bachelard et Henri Bosco, tout en observant de loin ses personnages, qui existent sans lui, et en tenant à distance les mots, qui l'effraient vaguement. En outre, le " commis aux écritures " souffre d'un complexe d'infériorité: il serait un écrivain s'il ne s'ignorait pas délibérément et ne s'estimait pas indigne des grandes figures qui peuplent son panthéon personnel, dont Hemingway est le prototype, qui le domine de toute sa superbe. Que cache donc ce fameux "commis aux écritures ", cet éternel adolescent qui s'est donné à la littérature comme d'autres se lancent en religion, qu'on retrouve d'un roman à l'autre de Jacques Poulin, dissimulé sous des pseudonymes qui ne trompent finalement personne? Ce personnage en perpétuel devenir serait-il symptomatique d'une culture et d'une société qui se cherchent? Sans doute. Mais ne semblerait-il pas surtout représentatif d'une littérature «qui se fait» en se prenant elle-même pour objet, tout en ne manquant jamais de se situer en creux devant le monde des écrivains de génie, sorte d'univers parallèle qui la transcende et qu'elle présume inaccessible? De plus, il est à se demander si le système hiérarchique qui se trouve établi par Jacques Poulin au moyen du personnage du * commis * et de la figure fantasmatique de l' "écrivain " ne dissimulerait pas, en la justifiant, une autre hiérarchie, d'ordre générique celle-là, et qui traduirait non plus seulement l'état présumément germinatif de l'œuvre de Jacques Poulin, mais aussi les difficultés rencontrées par le roman québécois dans le processus de son affirmation en tant que genre littéraire, en regard 
de la poésie notamment. Le romancier québécois serait-il dans son essence un commis aux écritures?

Le cœur de la baleine bleue peut nous éclairer sur ce point; d'une part, il s'agit là sans doute du roman le plus "poétique" de Jacques Poulin, dans le sens que la poésie y occupe une place importante, exerçant son influence autant sur le contenu de l'œuvre que sur sa forme. Ce roman constitue de surcroît une remarquable méditation sur l'œuvre en train de se faire, nous permettant de saisir à vif les interactions entre les genres littéraires et d'assister en quelque sorte à la naissance du langage romanesque, qui se dégage progressivement du langage poétique pour s'affirmer bientôt dans toute sa richesse et, paradoxalement, dans son dénuement absolu.

Le cœur de la baleine bleue 1 s'ouvre sur le récit autobiographique d'un personnage fictif, qu'un roman tout aussi fictif, inspiré de l'enfance de ce personnage, vient compléter. $\mathrm{Au}$ lendemain d'une transplantation cardiaque, Noël s'est attaqué à l'écriture d'un roman. Au départ, ce roman devait raconter l'histoire de Jimmy, un garçon qui séquestre une fillette dans le but de la violer, "pour avoir la paix" (p. 40), comme il l'affirme lui-même sans ambages; mais le déroulement de cette histoire, que l'auteur voyait comme une * aventure brutale» (p. 83), se trouve bientôt infléchi par l'étrange douceur qui semble avoir envahi Noël depuis son opération, douceur qu'il attribue au cœur de jeune fille qu'on lui a donné et qui vient former comme un écran entre sa conscience et les personnages qu'il avait inventés, d'où provient sans doute son impuissance devant l'œuvre à venir: " Javais la gorge serrée et je sentais bouillonner toutes ces choses en moi sans pouvoir les exprimer; à la surface, c'était une nappe de douceur, une mer d'huile qui bloquait tout * (p. 92). Tandis qu'il s'enfonce de plus en plus dans son monde intérieur, à la poursuite d'un sujet qui lui échappe sans cesse, tandis qu'il se lance dans une quête tâtonnante du rapport obscur qu'il perçoit confusément entre la douceur qui l'habite et la mort qui l'attend, sa vie conjugale s'en va à la dérive. C'est ainsi que sa femme Élise, qu'il avait délaissée au profit de son roman, l'abandonne à son tour pour convoler avec Bill, un joueur de hockey.

1 Coll, « Poche Québec ", n²5, Montréal, Leméac, 1987, 201 p. Les citations renvoient à cette édition. Le roman a d'abord paru en 1970 aux Éditions du Jour. 
Tout le drame de Noël vient du fait qu'en bon commis aux écritures, il entretient une relation ambiguë avec la société et le langage, qui lui sont toujours vaguement étrangers: la société où il doit évoluer appartient aux femmes comme Élise et aux joueurs de hockey, tandis que le langage demeure la chasse gardée des poètes et des écrivains. Lui se trouve coincé entre ces deux pôles: comme son malaise lui interdit simultanément d'agir sur la société et sur le langage, il se voit relégué en marge de la vie, dans un monde purement imaginaire où les souvenirs idéalisés de la première enfance se conjuguent à l'attrait de la mort pour se fondre finalement dans une douceur absolue, dans une immobilité totale où l'existence s'anéantit, ce qui le pousse à affirmer: « Je ne pourrai jamais vivre dans ce paysage parce que la vie, c'est l'agressivité n (p. 191).

Pour Noël, le cours de la vie ressemble donc à une gigantesque partie de hockey où il se trouve pris en tenaille entre des clans adverses qui l'ignorent. Les femmes comme Élise sont vaguement menaçantes, quand elles ne deviennent pas synonymes de chute, de damnation:

Son regard était trouble comme l'eau dormante d'un marais et, l'espace d'un instant, je crus voir au fond de ses yeux de longs doigts poilus qui remuaient comme des insectes. Je la regardais, fasciné; il me semblait que je glissais le long des parois humides d'un puits dont le fond m'attirait irrésistiblement (p. 28).

Quoi qu'il en soit, la femme n'évolue pas dans la même sphère que le commis aux écritures; Élise reproche ainsi à son mari son détachement à l'égard de la réalité: *Tu rêves toujours, toi. Tu passes ton temps à ça» (p. 14). Quant à Noël, il affirme: «Elle s'occupait des choses, et moi des rêves; il lui arrivait de me ramener sur terre assez brusquement * (p. 30). Dans cet envers du rêve et de l'enfance où se meut la femme, on retrouve aussi le joueur de hockey, qui symbolise quant à lui la virilité et la force qui font défaut au commis :

J'avais toujours pensé que les joueurs de hockey n'avaient pas d'enfance; je veux dire: qu'ils ne vivaient pas avec leur enfance, excepté un petit nombre, comme Frank Mahovlich et Bob Rousseau, qui trainaient encore une partie de leur enfance sur la patinoire (p. 89).

Tenu à l'écart du monde des humains, Noël n'en entretient pas moins un rapport problématique avec le langage et 
son métier de pseudo-écrivain, ne maîtrisant ni l'un ni l'autre. Tout lui échappe. Ainsi du langage, qui constitue en soi un univers transcendant où l'homme demeure toujours plus ou moins un étranger, puisqu'il est dans l'obligation de composer avec une réalité qui le dépasse: *Et si le langage n'était pas en nous? Et si c'était l'homme qui habitait dans le langage?» (p. 23). De la même façon, l'écriture devient pour Noël une activité ambiguë, engageant moins sa liberté qu'une certaine soumission à un destin qui lui est assigné: «Pourtant je continuais, je me sentais poussé à le faire. Je n'étais pas un véritable écrivain, mû par un besoin irrépressible de créer, d'exprimer ou de communiquer; cela ressemblait plutôt à une idée fixe " (p. 84). Comme son maître Henri Bosco, Noël n'écrit donc pas vraiment, il transcrit des visions, des hallucinations: * J'avais fini par m'en remettre à cet étranger, installé au creux de moi-même, qui voyait tout d'une autre manière, et la seule responsabilité que je m'attribuais encore, c'était d'être là, présent, prêt à transcrire les volontés parvenues jusqu'à moi » (p. 41). Condamné à l'écriture, jeté dans l'univers du langage, Noël s'interroge bientôt sur la précarité de son statut dans le monde: "Je n'étais ni heureux ni malheureux, puisque j'écrivais. Et même, je ne me sentais pas véritablement écrivain, puisque j'étais en vie» (p. 43). Ce mort-vivant qui hante simultanément la littérature et la terre des humains n'est donc finalement à l'aise nulle part: il n'est pas un écrivain puisqu'il est en vie, il n'est pas un humain puisqu'il écrit. Il se trouve relégué dans une zone intermédiaire, sorte de no man's land où prend racine sa vocation de proto-romancier: "Quand on écrit, on a toujours l'impression d'être endehors de tout; pour se sentir dans le coup on se raconte des histoires" (p. 137). Et encore n'est-il jamais vraiment à l'aise dans ce métier: "Je me demandais si on pouvait être aussi honnête en écrivant des histoires au lieu de s'en tenir à la stricte autobiographie» (p. 45).

Restent du moins les mots qui, en singularisant l'univers plutôt abstrait et transcendant du langage, représentent une source de fascination pour le commis aux écritures: «Les mots ont certainement une âme» (p. 144). Certes, une telle fascination n'est pas exempte d'une certaine angoisse devant les mots et le pouvoir qu'ils possèdent: «Et puis, j'écrivais péniblement. Je veux dire: j'écrivais peureusement, comme s'il y avait quelque chose d'inquiétant au bout des mots; comme si, au tournant d'une phrase, j'allais brusquement me trouver 
34

face à face avec je ne sais quoi de menaçant et d'irrémédiable " (p. 83-84). Néanmoins, c'est par le biais de cette fascination que le commis va se rapprocher imperceptiblement de l'écrivain, pour découvrir enfin, au terme de l'aventure que lui proposent les mots, son véritable statut, la place qui lui est assignée quelque part dans le monde: "On aurait dit que les mots constituaient en même temps la seule issue possible, une sorte d'initiation, un rite de passage comme certaines tribus primitives en faisaient subir aux adolescents qui prétendaient devenir des hommes * (p. 84).

Or, si les mots semblent constituer un parcours initiatique conduisant le commis aux écritures depuis l'indistinction jusqu'à l'âge d'homme, ils n'en représentent pas moins un danger de taille: celui de la dispersion, de l'égarement. En admettant que les mots aient une âme et qu'ils existent comme autant de micro-univers, le commis aux écritures ne risque-t-il pas de se perdre sur une route lui offrant une infinité de carrefours? C'est à ce stade que les poètes entrent en jeu. Dès le départ, Noël pressent qu'il existe un lien entre son aventure au fil des mots et le projet poétique d'André Breton, dont il cite une expression qui deviendra bientôt un des leitmotive du roman: * Partir pour le pôle intérieur de soimême* (p. 31). Selon Noël, les poètes peuvent percevoir « des réalités inconnues au commun des mortels ", parce qu'ils sont * plus près des choses * (p. 56). Au contraire, même s'il est séduit par les mots, le commis aux écritures reste toujours en marge des choses: «J'aimais que les gens écrivent sur les murs, sur les maisons, sur les trottoirs, dans la rue, partout. De toute façon j'aimais les mots. Ce qui m'échappait, c'était les rapports entre les choses * (p. 152). Comme ils sont dotés d'une existence autonome qui leur permet de * décoller* de l'objet, les mots forment eux aussi un écran entre la conscience de l'apprenti-romancier et le monde qui l'entoure, mais qu'en retour il ne parvient jamais à entourer au moyen de son mode d'expression; par conséquent, il semble condamné à demeurer dans le sillage du poète à qui est conféré, selon une esthétique assez baudelairienne, le rôle du phare, ou plutôt du précurseur, voire du prophète: «Sans pouvoir comprendre, je devinais que les poètes nous laissaient parfois derrière eux sur une route faiblement éclairée, comme celle que j'avais empruntée pour écrire mon histoire [...]» (p. 152). À l'origine du roman, on retrouvera donc souvent soit un poème, soit un vers, soit une image: 
- Vos romans commencent de quelle manière... Au début, je veux dire, il y a quoi?

- Le plus souvent c'est une image, dis-je. Mais il faut la laisser pourrir lentement (p. 21).

La poésie occupe ainsi une position stratégique dans l'univers littéraire: en un sens, elle est antérieure au genre romanesque, auquel elle imprime un élan vital en lui insufflant du sens. Par conséquent, doit-on s'étonner de retrouver, en exergue au Cour de la baleine bleue, ce vers de Pierre Morency: "C'est dans ma poitrine que j'écris * ${ }^{2}$ ? Il ne s'agit pas seulement d'annoncer, au moyen du vers, le sujet du roman, mais aussi de situer ce dernier dans le processus de sa propre genèse, en remontant au point zéro de l'écriture, au foyer originel d'où jaillit le discours romanesque. C'est d'ailleurs pourquoi le vers qui est cité en exergue nous renvoie nécessairement à son contexte immédiat, c'est-à-dire au poème qui l'englobe et dont il ne peut être tout à fait dissocié. En consultant le poème de Morency, on constate ainsi que certains des grands thèmes du roman de Poulin s'y profilent très manifestement. Considérons par exemple la complète solitude du commis aux écritures devant le monde des humains, que l'épisode du carnaval illustre admirablement: «Élise était sortie avec Bill pour assister à la course de canots. J'étais resté pour écrire [...] De temps en temps, ils dansaient comiquement. Il semblait faire très froid. Je revins à mon paysage. L'été. La douceur * (p. 100, 104). Comparons maintenant cette superbe solitude avec celle du poète: «Je sors très peu / je passe mes journées en dedans de mes côtes ». Un autre exemple: dans son voyage vers le pôle intérieur, le commis suit au fond les traces du poète; quand il raconte: «En suivant le fil de cette histoire, j'étais parti sans le savoir à la recherche de moi-même, je remontais à la source $[. .] ».(p .83)$, le commis n'accompagne-t-il pas, à distance, le poète qui écrivait: «Et quand je voyage je descends dans mon ventre/ au plus profond des voixn? De la même façon, l'écart qui prend place chez le commis entre le "cœur» et la raison ( «Je ne réfléchis pas beaucoup, presque jamais; les mots cheminent en moi et finissent par déboucher sur la lumière» - p. 145),

2 Ce poème, qui appartient aux Podmes de la vie déliée (1968), est reproduit dans Quand nous serons; poemes 1967-1978, Montréal, L'hexagone, 1988. p. $77-78$. 
semble trahir le malaise du poète devant la pensée rationnelle: «Rarement je monte jusque dans ma tête / je ne me reconnais pas dans ma tête . D'une certaine façon, il n'est donc pas exagéré d'affirmer que Le cour de la baleine bleue (1970) n'existerait pas vraiment sans les Poemes de la vie déliee (1968). D'ailleurs, si on se fie rétrospectivement à l'esthétique élaborée par Jacques Poulin au fil de ses œuvres, on constate que ses romans, quand bien même ils s'informent mutuellement, l'un annonçant l'autre, ne manquent jamais de se situer dans une lignée qui les dépasse infiniment: consciemment ou inconsciemment, ils appartiennent à un continuum littéraire où les catégories génériques semblent en étroite communication. Par exemple, dans Volkswagen blues (1984), Jack Waterman explique à la Grande Sauterelle:

II ne faut pas juger les livres un par un. Je veux dire: il ne faut pas les voir comme des choses indépendantes. Un livre n'est jamais complet en lui-même; si on veut le comprendre, il faut le mettre en rapport avec d'autres livres, non seulement avec les livres du même auteur, mais aussi avec des livres écrits par d'autres personnes. Ce que l'on croit être un livre n'est la plupart du temps qu'une partie d'un autre livre plus vaste auquel plusieurs auteurs ont collaboré sans le savoir. ${ }^{3}$

On peut donc pressentir de quelle manière le langage romanesque parvient à interférer avec le langage poétique sans le dénaturer, mais on peut se demander comment en retour l'énoncé poétique, qu'il s'agisse du vers cité ou de son contexte immédiat, ne limite-t-il pas l'expression romanesque ? À la rigueur, le poème n'en viendrait-il pas à encercler le roman, à le priver d'une certaine liberté? C'est ce que semble dénoter la place qu'occupe, dans Le cœur de la baleine bleue, le poème « Cage d'oiseau " de Saint-Denys Garneau. Si le vers de Pierre Morency annonce la thématique du roman de Jacques Poulin tout en donnant une certaine coloration à son décor imaginaire, le poème de Saint-Denys Garneau structure quant à lui la progression même du roman; en fixant le destin tragique du commis aux écritures, comme l'autorise d'ailleurs le rôle prophétique qui est dévolu aux poètes, tout en justifiant les nombreux rapports métaphoriques qui sont établis par le narrateur entre le cœur qu'on lui a donné et un

3 Jacques Poulin, Volkswagen blues, coll. Littérature d'Amérique n, Montréal, Québec/Amérique, 1989, p. 169. 
oiseau qu'on aurait emprisonné. C'est justement la connaissance du poème de Saint-Denys Garneau qui donne tout son sens à l'aventure de Noël et qui permet au lecteur d'appréhender véritablement celle-ci, depuis le moment de la transplantation du cœur ( $\star$ Je suis une cage d'oiseau / Une cage d'os / Avec un oiseau *) jusqu'à sa libération finale ( II aura mon âme au bec $\bowtie$ ) en passant par l'angoisse terrible du rejet ("L'oiseau dans sa cage d'os / C'est la mort qui fait son nid n; "C'est un oiseau tenu captif / La mort dans ma cage d'os w). Á ce sujet, Élise fait d'ailleurs remarquer à Noël: «C'est comme si tu te dévorais toi-même» (p. 50).

La poésie réalise ainsi une percée assez singulière dans l'univers du roman, en ordonnant à l'avance le déroulement et le dénouement de l'intrigue; dans Le coeur de la baleine bleue, la poésie est d'autant plus souveraine qu'elle prend le visage du destin. Noël en semble d'ailleurs très conscient, puiqu'il a peur de se rappeler la fin du poème de Saint-Denys Garneau: «La suite du poème ne venait pas. Javais l'impression d'échapper à un danger obscur, comme si ma mémoire avait rejeté dans l'oubli ce qui me menaçait» (p. 21). Il ne s'agit donc pas là exactement d'une mise en abyme: le poème ne vient pas simplement appuyer le discours romanesque en le dédoublant dans un autre registre de l'expression, ce qui reviendrait à négliger la fonction exploratoire qui est dévolue au genre poétique. Non, la poésie se situe plutôt au fondement même de toute réalité, au moment précis de l'avènement du réel. Pour Noël, tout se passe comme si la poésie régnait sur le cours des choses, se manifestant parfois dans toute sa plénitude: "Alors les derniers vers de Saint-Denys Garneau me revinrent en mémoire; ils parlaient de l'oiseau dans sa poitrine et j'entendis, plus distinctement que si Élise me les récitait elle-même [...] ( (p. 50).

Remarquons enfin qu'en marge de cette influence déterminante de la poésie sur la thématique et le déroulement du roman, la chanson joue aussi un rôle important dans Le cour de la baleine bleue, en s'y déployant comme une toile de fond, ou plus exactement comme une tapisserie sonore où seraient tissés des harmoniques venant appuyer la ligne mélodique tracée par la coexistence des poèmes et du récit de l'homme au cœur de jeune fille: "J'aimais les chansons. Depuis l'opération, j'avais toujours une chanson dans la tête * (p. 9). Tout le roman oscille ainsi de la poésie à la chanson, de sorte 
38

qu'on se retrouve bientôt devant une œuvre qui, certes, doit beaucoup aux genres littéraires présidant à sa naissance et son évolution, mais dont la légitimité et l'autonomie génériques se voient comme diminuées au profit des langages qui l'encombrent. A ce stade, nous devons nous référer à Mikhail Bakhtine qui oppose, dans Esthétique et théorie du roman, le langage du poète à celui du prosateur: si "le style poétique est conventionnellement aliéné de toute action réciproque avec le discours d'autrui ${ }^{4}$, * le prosateur, quant à lui, tente de dire dans le langage d'autrui ce qui le concerne personnellement ${ }^{5}$. C'est d'ailleurs pourquoi le langage du poète, dans son cheminement vers l'objet, rencontre moins de résistance que le langage du prosateur, pour qui « l'objet est le point de convergence de voix diverses, au milieu desquelles sa voix aussi doit retentir ${ }^{6}$, et que Noël peut affirmer que les poètes sont «plus près des choses». Bien que tout prosateur se trouve dans l'obligation d'assumer cette plurivocalité, qui crée pour lui " un fond indispensable, hors duquel ne sont ni saisissables, ni "résonnantes" les nuances de sa prose littéraire*, il se doit aussi de choisir, dans la multitude des langages qui s'imposent à lui, un langage qui lui isera propre: « Toujours et partout, à tous les âges de la littérature historiquement connus, la conscience littéraire active découvre des langages et non un langage. Elle se trouve devant la nécessité du choix d'un langage "?. Or, n'est-ce pas l'ambition secrète du commis aux écritures que celle de trouver enfin sa place dans le langage, de découvrir son langage, pour s'élever au rang de l'écrivain?

De là viendrait la nécessité qu'a ressentie Jacques Poulin de "greffer», au récit premier que forme l'autobiographie fictive de Noël, un deuxième récit, consacré celui-là à la rencontre avec Charlie, une jeune fille qui va jouer, auprès du commis aux écritures en quête de son pôle intérieur, un rôle initiatique très apparent, qui ne manque pas de rappeler celui tenu par la Grande Sauterelle dans Volkswagen blues et, dans une autre perspective, la place occupée par les mots dans Le cœur de la baleine bleue. Charlie sera donc une

4 Mikhaïl Bakhtine, Esthétique et théorie du roman, coll. $\times$ Tel $», n^{\circ} 120$, Paris, Gallimard, 1987, p. 107.

5 lbid., p. 109.

6 lbid., p. 102.

7 lbid., p. 116. 
création pure du langage, tandis que le récit relatant sa venue, qui regroupe certaines des pages les plus remarquables qu'ait écrites Jacques Poulin, constituera une épure de roman $^{8}$.

À première vue, ce récit peut sembler assez énigmatique: il apparait tout à coup, au milieu de l'autobiographie de Noël, sans lien manifeste avec ce qui le précède, au moyen d'une phrase très simple qu'encadrent des astérisques: “C'est une histoire de cœur» (p. 17). Ici, nulle nécessité d'aller de la poésie vers le roman, nul besoin de faire appel à un genre extrinsèque: le roman s'impose immédiatement, il naît littéralement, littérairement oserait-on dire, sous les yeux du lecteur, comme un cœur qui bat pour la première fois, qui est encore comme suspendu au souffle fragile de la vie, mais qui affirme bientôt sa cadence: «Une histoire de cœur entre moi et le vieux Québec" (p. 19), jusqu'à trouver finalement son propre rythme: ${ }^{\circ}$ C'est une histoire de cœur entre moi et le vieux Québec. Je suis assis dans les marches de la librairie Garneau, pas devant l'entrée principale, mais devant la section des livres pour enfants $»$ (p. 24). Des trois récits qui composent le roman de Jacques Poulin, c'est d'ailleurs le seul qui se trouve écrit au présent, comme si le roman n'avait justement pas besoin de passé pour exister, comme s'il se suffisait enfin à lui-même, se situant non seulement en marge des autres genres, mais aussi en marge du temps: on ne retrouve en effet, dans le récit consacré à Charlie, aucun indice permettant de situer clairement un ordre chronologique, puisque le temps n'a plus d'importance au regard d'une œuvre qui jaillit dans son innocence primitive.

C'est donc ce récit énigmatique qui va s'imposer peu à peu, à l'intérieur du Cœur de la baleine bleue, comme le véritable roman, en faisant succéder, au réalisme caractérisant l'autobiographie de Noël et aux résonances psychologiques du roman fictif consacré à Jimmy, un idéalisme presque désincarné, un «angélisme » qui est somme toute beaucoup plus conforme au ton et à la manière de Jacques Poulin. Ne provenant plus de la poésie, mais libre néanmoins d'aller vers elle, le roman a trouvé en quelque sorte sa voie; il a atteint,

8 Consulter à ce sujet l'excellent article de Gabrielle Poulin, « Le ccour de la baleine bleve de Jacques Poulin; un roman piégé ", Relations, Montréal, mai 1972, p. 154-155. 
40

lui aussi, le pôle intérieur. En réalisant une épure de roman qui émerge, comme un cœur qui commence à battre, de la poésie, de la chanson, de l'autobiographie, le * commis aux écritures " a fait un premier pas vers la condition d'écrivain, se liberrant du même coup de sa propre cage. Le roman se termine d'ailleurs sur ces phrases: « J'ai une chanson dans la tête mais je ne trouve pas le titre. Non, c'est plutôt comme le chant d'un oiseau. Un oiseau en liberté» (p. 201). 